\title{
Diarrhea and delirium from naltrexone-precipitated opioid withdrawal
}

\author{
Isabelle Miles, MD*; Andrew Kestler (D, MD, MBA, MSc ${ }^{*}$; Frank X. Scheuermeyer, MD, MHSc ${ }^{* \dagger}$
}

Keywords: Addiction, substance misuse, toxicology

\section{INTRODUCTION}

Naltrexone is a competitive opioid antagonist ${ }^{1}$ that is first-line therapy for alcohol use disorder and alternative therapy for opioid use disorder. Should a patient actively using regular opioids ingest naltrexone, rapid severe opioid withdrawal might take place (clinical opioid withdrawal scale $[\mathrm{COWS}]>36$ ). ${ }^{2-4}$ We report two such cases, both likely due to diverted naltrexone.

\section{Case 1}

A 50-year-old woman was transported by ambulance (EMS) to an urban emergency department (ED) with sudden profound agitation and profuse watery diarrhea but no further history. Her heart rate was 150 beats per minute, temperature $37.4^{\circ} \mathrm{C}\left(99^{\circ} \mathrm{F}\right)$, and blood pressure 130/80 $\mathrm{mm} \mathrm{Hg}$. There were no signs of trauma, and she had sluggish 4-mm pupils bilaterally with normal respiratory effort. She was given $20 \mathrm{mg}$ of midazolam intravenously for agitation. Her provincial pharmanet indicated that she was only prescribed daily methadone $100 \mathrm{mg}$, including that morning. Her agitation and watery diarrhea worsened, and she required sedation with $40 \mathrm{mg}$ of midazolam and $20 \mathrm{mg}$ of loxapine over the next 4 hours; she was administered $4 \mathrm{~L}$ of normal saline to account for gastrointestinal losses.

All blood testing was normal, and her urine tested positive for methadone and benzodiazepines. Head computed tomography (CT) was normal. The patient additionally required $40 \mathrm{mg}$ of midazolam over the next 24 hours, generally in 5-mg boluses every 2 hours, as well as saline at $200 \mathrm{~mL} /$ hour. She remained in the ED overnight, and 25 hours post arrival she normalized. Shortly after ingesting methadone the day before, she bought a single pill from a new supplier, assumed it to be diazepam, and remembered little except profuse diarrhea. An addictions physician showed her a naltrexone $50 \mathrm{mg}$ pill and she confirmed that she had taken that pill. She was discharged uneventfully with successful follow-up at the addictions clinic.

\section{Case 2}

A 45-year-old male was transported to an urban community ED by means of EMS with sudden onset of agitation, severe diarrhea, vomiting, diaphoresis, and diffuse musculoskeletal pain shortly after taking an unknown pill. He was taking daily witnessed ingestion methadone for opioid use disorder. Vital signs and blood testing were normal. His COWS was 38, and soon after his arrival, he volunteered that the culprit pill had not been prescribed, but provided by a family member and called naltrexone. Fluid losses were treated with a total of $3 \mathrm{~L}$ of normal saline over the next 3 hours, and he was given lorazepam $2 \mathrm{mg}$ orally to treat both agitation and withdrawal symptoms. He was admitted to an internal medicine ward overnight for ongoing symptom management before uneventful discharge, with primary care follow-up 2 days later.

\section{DISCUSSION}

Naltrexone (half-life 4 hours) is liver-metabolized to 6B-naltrexol (half-life 13 hours), ${ }^{1}$ and excreted in the

From: *Department of Emergency Medicine, St Paul's Hospital and the University of British Columbia, Vancouver, BC; and the †Center for Health Evaluation and Outcome Science, University of British Columbia, Vancouver, BC.

Correspondence to: Dr. Frank X. Scheuermeyer, 1081 Burrard Street, Vancouver, BC V6Z 1Y6 Canada; Email: frank.scheuermeyer@gmail.com

(C) Canadian Association of Emergency Physicians

CJEM 2020;22(1):121-122

DOI 10.1017/cem.2019.425 
urine. Both are competitive reversible opioid receptor antagonists, strongest at the mu receptor (where they outcompete other opioids, including methadone), less strong at the kappa receptor, and weakest at the delta receptor. The combined effect is to lower mesolimbic dopamine concentration, ${ }^{1}$ reducing euphoria from opioid and alcohol use and decreasing cravings. It is firstline pharmacologic therapy for alcohol use disorder ${ }^{1}$ and possible alternative therapy for select patients with opioid use disorder. While a once-monthly depot version is approved in the United States, ${ }^{1}$ the only Canadian formulations are daily $25-$ and $50-\mathrm{mg}$ tablets.

Naltrexone use may become more common for alcohol use disorder, and emergency physicians need to be aware of the consequences of its inappropriate ingestion. Because this is a competitive antagonist whose effects will persist at least 24 hours, patients generally require 7 days or more without opioids before its initiation ${ }^{1-3}$; otherwise, administration can result in profound opioid withdrawal (COWS $>36$; in the first case, the score was 41 ) for $24-48$ hours, and this can be lifethreatening. ${ }^{2-4}$

The diagnosis is exclusionary, as ominous metabolic, structural, traumatic, and additional toxicological entities also require consideration. ${ }^{2-4}$ Confirmatory serum (quantitative) and urine (qualitative) tests may not be available in all settings and results typically take days to return, outside the time frame for management.

In 132 patients at a single Iranian ED, nearly all $(96.2 \%)$ had agitation, while approximately one-quarter each had nausea, vomiting, abdominal pain, and onesixth had diarrhea. Ominously, the authors attributed a $32 \%$ "partial recovery" to a high incidence of aspiration pneumonia; one patient had a fatal myocardial infarction during the recovery phase. ${ }^{3}$ An Australian ED described 18 patients considered to have primary gastrointestinal symptoms, treated chiefly with crystalloid fluid and antiemetics over a mean 11-hour length of stay. Fourteen patients had primarily severe agitation, treated with a mean dose of $211 \mathrm{mg}$ of diazepam or midazolam over a mean length of stay of 23 hours; two patients required intubation for airway compromise. ${ }^{2}$
Lack of evidence-based guidelines adds to management challenges. Agitation can be managed with benzodiazepines. Nausea and vomiting are treated with antiemetics and airway protection is paramount. ${ }^{2,3}$ Fluid losses should be estimated and replaced with crystalloid to maintain blood pressure and urine output. Musculoskeletal pain should be managed with nonopioid analgesia, and withdrawal can be treated with clonidine $0.1-0.2 \mathrm{mg}$ every 6 hours. $^{2}$ In consultation with addiction specialist or toxicologist, opioids with strong affinity for the mu receptor such as hydromorphone $e^{5}$ can be considered for withdrawal management. While mean hospitalization time has been reported as 18 hours, $^{2}$ far longer stays occur if substantial sedation or intubation is required. ${ }^{2-4}$

\section{CONCLUSION}

For patients with regular, and recent, opioid use, severe agitation or other manifestations of opioid withdrawal should prompt physicians to consider naltrexoneprecipitated withdrawal, while providing supportive care and ruling out other aetiologies.

Acknowledgements: F.S. conceived the idea, and I.M. and F.S. provided the cases. F.S. drafted the manuscript with critically important revisions from I.M., and assistance from A.K.

\section{REFERENCES}

1. Anton RF. Naltrexone for the management of opioid dependence. N Engl 7 Med 2008;359:715-721.

2. Armstrong J, Little M, Murray L. Emergency department presentations of naltrexone-accelerated detoxification. Acad Emerg Med 2003;10:860-866.

3. Hassanian-Moghaddam H, Afzali S, Pooya A. Withdrawal symptom caused by naltrexone in opioid abusers. Hum Exp Toxicol 2014;33:561-567.

4. Wrightman RS, Nelson LS, Lee JD, Fox LM, Smith SW. Severe opioid withdrawal precipitated with Vivitrol. $A m \mathcal{F}$ Emerg Med 2018;36:1128e1-1128.e2.

5. Volpe DA, Tobin G, Mellon RD, et al. Uniform assessment and ranking of opioid mu receptor binding constants for selected opioid drugs. Regul Toxicol Pharmacol 2011;59:385-390. 